Frank Rackwitz (Hrsg.)

Entwicklungen in der Bodenmechanik, Bodendynamik und Geotechnik 


\section{Entwicklungen in der Bodenmechanik, Bodendynamik und Geotechnik}

Festschrift

zum 60. Geburtstag von

Univ.-Professor Dr.-Ing. habil. Stavros A. Savidis

Herausgegeben von

Dr.-Ing. Frank Rackwitz

曼 Springer 


\author{
Dr.-Ing. Frank Rackwitz \\ Technische Universität Berlin \\ Institut für Bauingenieurwesen \\ Gustav-Meyer-Allee 25 \\ 13355 Berlin \\ frank.rackwitz@tu-berlin.de
}

Bibliografische Information der Deutschen Bibliothek

Die Deutsche Bibliothek verzeichnet diese Publikation in der Deutschen Nationalbibliografie;

detaillierte bibliografische Daten sind im Internet über http://dnb.ddb.de abrufbar.

$\begin{array}{ll}\text { ISBN } 10 & 3-540-27425-1 \\ \text { ISBN } 13 & 978-3-540-27425-4\end{array}$

Dieses Werk ist urheberrechtlich geschützt. Die dadurch begründeten Rechte, insbesondere die der Übersetzung, des Nachdrucks, des Vortrags, der Entnahme von Abbildungen und Tabellen, der Funksendung, der Mikroverfilmung oder Vervielfältigung auf anderen Wegen und der Speicherung in Datenverarbeitungsanlagen, bleiben, auch bei nur auszugsweiser Verwertung, vorbehalten. Eine Vervielfältigung dieses Werkes oder von Teilen dieses Werkes ist auch im Einzelfall nur in den Grenzen der gesetzlichen Bestimmungen des Urheberrechtsgesetzes der Bundesrepublik Deutschland vom 9. September 1965 in der jeweils geltenden Fassung zulässig. Sie ist grundsätzlich vergütungspflichtig. Zuwiderhandlungen unterliegen den Strafbestimmungen des Urheberrechtsgesetzes.

Springer ist ein Unternehmen von Springer Science+Business Media

springer.de

(C) Springer-Verlag Berlin Heidelberg 2006

Printed in Germany

Die Wiedergabe von Gebrauchsnamen, Handelsnamen, Warenbezeichnungen usw. in diesem Buch berechtigt auch ohne besondere Kennzeichnung nicht zu der Annahme, dass solche Namen im Sinne der Warenzeichen- und Markenschutz-Gesetzgebung als frei zu betrachten wären und daher von jedermann benutzt werden dürften. Sollte in diesem Werk direkt oder indirekt auf Gesetze, Vorschriften oder Richtlinien (z. B. DIN, VDI, VDE) Bezug genommen oder aus ihnen zitiert worden sein, so kann der Verlag keine Gewähr für die Richtigkeit, Vollständigkeit oder Aktualität übernehmen. Es empfiehlt sich, gegebenenfalls für die eigenen Arbeiten die vollständigen Vorschriften oder Richtlinien in der jeweils gültigen Fassung hinzuzuziehen.

Umschlaggestaltung: deblik, Berlin

Satz: Digitale Druckvorlage des Herausgebers

Druck: Mercedes-Druck, Berlin

Verarbeitung: Stein +Lehmann, Berlin

Gedruckt auf säurefreiem Papier $\quad 68 / 3020 / m \quad-543210$ 


\section{Vorwort}

Der 60. Geburtstag von Herrn Univ.-Professor Dr.-Ing. habil. Stavros A. Savidis diente seinen Schülern, Mitarbeitern und Fachkollegen als Anlass, sein mehr als 35-jähriges Schaffen als Forscher, Ingenieur und Lehrer auf den Gebieten Bodenmechanik, Bodendynamik und Geotechnik mit einer Festschrift zu würdigen.

In 26 Fachaufsätzen werden Themen aus den Bereichen theoretische und experimentelle Bodenmechanik, Bodendynamik und dynamische Boden-Bauwerk Interaktion, Geotechnik und Spezialtiefbau sowie Verkehrswegebau und Umweltgeotechnik behandelt. Ein Teil der Beiträge beschäftigt sich mit der Darstellung neuer wissenschaftlicher Erkenntnisse und der Formulierung neuer Fragestellungen und Lösungsansätze. Bei anderen Aufsätzen steht die Umsetzung der Theorie in die Baupraxis im Vordergrund und es werden konkrete Beispiele aus der Praxis vorgestellt und diskutiert. Das Buch richtet sich daher sowohl an Lehrende und Forschende als auch an Ingenieure, die auf diesen Gebieten arbeiten. Durch die Bandbreite der behandelten Themen werden sich auch viele Interessierte aus angrenzenden Fachgebieten angesprochen fühlen und Anregungen für die eigene Arbeit finden.

An dieser Stelle sei den Autoren für ihre Mühen bei der Ausarbeitung der Fachbeiträge herzlich gedankt. Beim Springer-Verlag und seinen Mitarbeitern möchte ich mich für die unkomplizierte und sehr zügige Vorbereitung und Begleitung des Drucks bedanken, sowie für die stets freundliche und kompetente Unterstützung bei der Herausgabe dieses Buches. Nicht zuletzt danke ich in diesem Zusammenhang auch Herrn cand.-ing. Patrick Herbke für die tatkräftige Mithilfe bei der Herausgebertätigkeit.

F. Rackwitz 


\section{Univ.-Professor Dr.-Ing. Stavros A. Savidis zum 60.Geburtstag}

Am 11. September 2004 vollendete Univ.-Professor Dr.-Ing. habil. Stavros Savidis, Ordinarius für Grundbau und Bodenmechanik an der Technischen Universität Berlin, sein 60. Lebensjahr. Professor Savidis wurde in Athen als Sohn des Juristen Aristophanis Savidis und seiner Ehefrau Filia geboren. Nach dem Besuch der Volksschule in Athen erlernte er bereits frühzeitig die Deutsche Sprache, denn er besuchte das Doerpfeld-Gymnasium, die Deutsche Schule in Athen, wo er 1962 sein Abitur erlangte. Anschließend bestand er als Bester unter ca. 1000 Bewerbern die Aufnahmeprüfung für ein Studium an der Technischen Hochschule Athen, wo er von 1962 bis 1967 Bauingenieurwesen studierte und wiederum als Jahrgangsbester abschloss. Im Oktober 1967 ging er als Stipendiat des DAAD an das von Professor Preß geleitete Institut für Wasserbau und Wasserwirtschaft an der Technischen Universität Berlin. Nach dem erfolgreichen Abschluss des gleichzeitig begonnenen Aufbaustudiums an der Fakultät für Bauingenieurwesen erfüllte er alle Voraussetzungen für die Zulassung zur Promotion an der TU Berlin. Infolgedessen wurde er im August 1968 als Wissenschaftlicher Assistent am Lehrstuhl und Institut für Grundbau und Bodenmechanik eingestellt, welches der Begründer der Grundbaudynamik, Professor Hans Lorenz, leitete. Mit der ihm eigenen außergewöhnlichen Energie und Akribie begann Professor Savidis unmittelbar nach seiner Anstellung die Arbeit an der von seinem Doktorvater vorgeschlagenen Dissertation mit dem Thema: „Die Wirkung des Viertelraumes auf die stationären erzwungenen Schwingungen eines Streifenfundamentes“. Nach nicht einmal zweieinhalb Jahren absolvierte er in der Folge im Januar 1971 mit dem Gesamturteil „Mit Auszeichnung“ mit Bravour seine mündliche Doktorprüfung. Es folgte im Dezember 1972 die Ernennung zum Assistenzprofessor für das Fachgebiet „Bodendynamik“, im Alter von 28 Jahren. Nach dem erfolgreichen Abschluss des Habilitationsverfahrens erlangte Professor Savidis 1977 die Lehrbefähigung für das Lehrgebiet „Grundbaudynamik und Bodenmechanik“" und es wurde ihm die Lehrbefugnis für dieses Fach an der TU Berlin verliehen. Im Jahr 1978 folgte er einem Ruf als ordentlicher Professor für Grundbau und Bodenmechanik an die neugegründete Technische Universität Xanthi in Griechenland. An der TU Berlin nahm er 1979 parallel zu den Aufbauarbeiten in Xanthi einen Ruf als C2Professor für das Fachgebiet Grundbaudynamik an. Die Professur in Berlin gab er 1983 auf, weil er als Rektor der TU Xanthi gewählt wurde und dieses Amt - 1986 erfolgte eine Wiederwahl - einen besonders intensiven Einsatz in Griechenland erforderte. 
Seiner Wahlheimat Berlin blieb er dennoch stets eng verbunden. So folgte er schließlich zum Sommersemester 1987 einem Ruf als Ordinarius für Grundbau und Bodenmechanik an der TU Berlin, als Nachfolger von Professor Müller-Kirchenbauer.

Die wissenschaftliche Tätigkeit von Professor Savidis ist wesentlich durch seine frühe Konzentration auf das Thema Bodendynamik geprägt, da er die Bedeutung dieser jungen Wissenschaftsdisziplin für die Baupraxis erkannte und sich für die entsprechende Berücksichtigung der Bodendynamik in Lehre und Forschung engagierte. Inzwischen mehr als 150 Veröffentlichungen auf den Teilgebieten analytische und numerische Methoden der elastischen und nichtlinearen Bodendynamik sowie der dynamischen Boden-Bauwerk Interaktion und darüber hinaus Arbeiten in den Bereichen Spezialtiefbau und Umweltgeotechnik zeugen von seiner langjährigen intensiven wissenschaftlichen Arbeit.

Beleg des hohen wissenschaftlichen Ansehens von Professor Savidis ist seine Wahl zum Fachgutachter der DFG im Jahr 1998 und seine Wiederwahl im Jahr 2003. Daneben ist er seit vielen Jahren Gutachter weiterer Institutionen zur Förderung der Wissenschaft, wie DAAD, BMBF und Volkswagenstiftung. Gleichzeitig bringt Professor Savidis seine Fachkenntnisse und Erfahrungen in zahlreiche Fachgremien, Organisationen und Normenausschüsse ein. So war er von 1996 bis 2004 Vorsitzender der Deutschen Gesellschaft für Erdbebeningenieurwesen und Baudynamik (DGEB) und ist seit 1992 Vorsitzender des Arbeitskreises 1.4 Baugrunddynamik der Deutschen Gesellschaft für Geotechnik (DGGT).

Hervorzuheben sind ebenfalls die vielen internationalen Kontakte von Professor Savidis zu Fachkollegen weltweit. Insbesondere diese beeindruckende Internationalität veranlasste 1985 den damaligen Präsidenten der TU Berlin dazu, Professor Savidis für die Verleihung des Max-Planck Forschungspreises für Internationale Kooperationen vorzuschlagen. In den Jahren 1989 und 1994 veranstaltete Professor Savidis in Berlin die vielbeachteten Internationalen Tagungen zum Erdbebengerechten Bauen - ERCAD.

Bislang mehr als 200 betreute Diplomarbeiten sowie 20 Erstgutachten abgeschlossener Dissertationen und die Begutachtung von drei abgeschlossenen Habilitationen, von denen heute zwei als Universitätsprofessoren und weitere fünf als Fachhochschulprofessoren wirken, stehen für seine umfangreiche Förderung des wissenschaftlichen Nachwuchses.

Im Rahmen seiner langjährigen Tätigkeiten an den Technischen Universitäten in Berlin und Xanthi engagierte sich Professor Savidis stets besonders aktiv in der akademischen Selbstverwaltung. So ist er beispielsweise, nach seiner Rektorentätigkeit in Xanthi, seit 1995 in fünf aufeinanderfolgenden Wahlperioden abwechselnd als Dekan bzw. Pro-Dekan an der TU Berlin tätig.

Neben seiner wissenschaftlichen und universitären Tätigkeit arbeitet Professor Savidis seit langem auch erfolgreich in der Praxis. Er war als selbständiger Beratender Ingenieur und für 
Professor Lorenz und Professor Müller-Kirchenbauer auf den Gebieten Grundbau und Bodendynamik gutachterlich tätig. In Griechenland war er Berater am Ministerium für Raumordnung und Umweltschutz sowie Vizepräsident des Staatlichen Instituts für Ingenieur-Seismologie und Aseismische Konstruktionen (ITSAK). Seit 1990 ist Professor Savidis als Geschäftsführender Gesellschafter des Ingenieurbüros GuD Geotechnik und Dynamik Consult GmbH in der Ingenieurberatung und als Gutachter für bodenmechanische, bodendynamische und grundbauliche Projekte für öffentliche und private Bauherrn im Inund Ausland erfolgreich tätig.

Das lange Zeit zu kurz gekommene private Leben trat 1993 durch die Heirat mit Georgia Alexandropoulou und 1999 mit der Geburt der Tochter Stephania in den Vordergrund.

$\mathrm{Zu}$ Ehren des 60. Geburtstages von Professor Savidis fand am 19. November 2004 ein FestKolloquium an der TU Berlin statt. Mehr als 150 Gäste folgten der Einladung nach Berlin und erlebten u.a. die Grußworte des Präsidenten der TU Berlin, Professor Kutzler, und eine würdige Laudatio von Professor Katzenbach. Den fachlichen Teil des Kolloquiums bildeten acht Beiträge von Fachkollegen aus China, Deutschland, Griechenland, Japan und den USA - eindeutiger Beleg der ausgezeichneten Reputation im In- und Ausland.

Zur Würdigung der Verdienste von Professor Savidis haben 50 Fachkollegen, Wegbegleiter und ehemalige Doktoranden aus dem In- und Ausland insgesamt 26 aktuelle Beiträge aus den Bereichen Bodenmechanik, Bodendynamik und Geotechnik verfasst, die in dem vorliegenden Buch erscheinen.

Seine Schüler, Mitarbeiter, Freunde und Kollegen wünschen dem Jubilar beste Gesundheit, Freude und Glück sowie weiterhin Schaffenskraft und Erfolg und nicht zu vergessen die notwendige Zeit für Erholung und Entspannung im Kreis seiner Familie.

\section{F. Rackwitz}




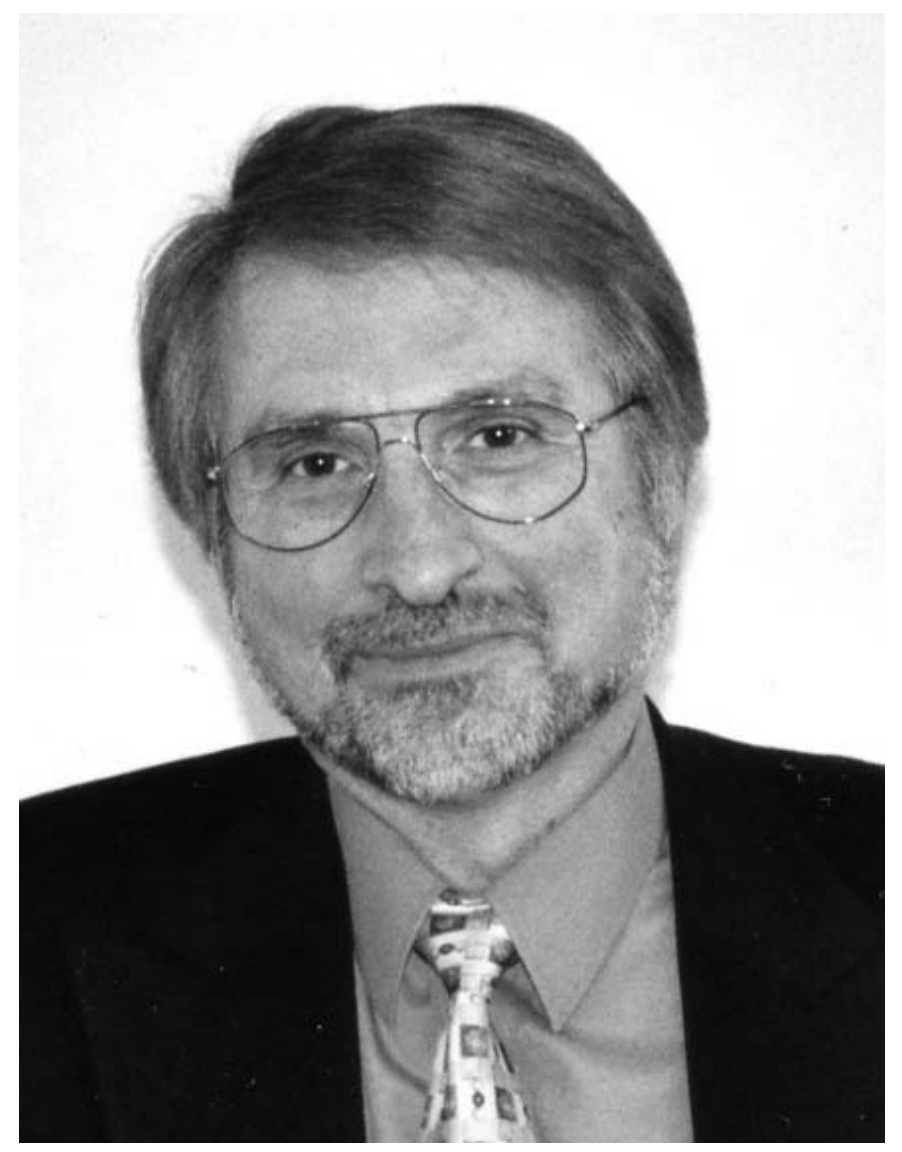

Univ.-Prof. Dr.-Ing. S. A. Savidis (Foto: TU Berlin/Pressestelle) 


\section{Autoren}

Anastasopoulos, Ioannis, M.Sc., National Technical University of Athens, Faculty of Civil Engineering, 42, Patission St., GR 10682 Athen

Baeßler, Matthias, Dipl.-Ing., Bundesanstalt für Materialforschung und -prüfung, Fachgruppe VII.2 - Ingenieurbau, Unter den Eichen 87, D 12205 Berlin

Bergmann, Stefan, Dr.-Ing., Elisenhof 1, D 15374 Müncheberg

Bode, Christopher, Prof. Dr.-Ing., Technische Fachhochschule Berlin, Fachbereich VIII, Luxemburger Straße 10, D 13353 Berlin

Borchert, Kurt-Michael, Prof. Dr.-Ing., GuD Geotechnik und Dynamik Consult GmbH, Dudenstraße 78, D 10965 Berlin

Eickenbrock, Christian, Dipl.-Ing., IGB Ingenieurgesellschaft mbH, Heinrich-Hertz-Str. 116, D 22083 Hamburg

Franke, Jörg, Dr.-Ing., IGB Ingenieurgesellschaft mbH, Heinrich-Hertz-Str. 116, D 22083 Hamburg

Gazetas, George, Prof. Dr., National Technical University of Athens, Faculty of Civil Engineering , 42, Patission St., GR 10682 Athen

Gerolymos, Nikos, Dr., National Technical University of Athens, Faculty of Civil Engineering, 42, Patission St., GR 10682 Athen

Gudehus, Gerd, o.Prof. Dr.-Ing., Universität Karlsruhe, Institut für Bodenmechanik und Felsmechanik, Lehrstuhl für Bodenmechanik und Grundbau, Engler-Bunte-Ring 14, D 76131 Karlsruhe

Hebener, Hans-Ludwig, Dipl.-Ing., GuD Geotechnik und Dynamik Consult GmbH, Dudenstraße 78, D 10965 Berlin

Hirschauer, Reinhold, Dr.-Ing., Nehringstr. 34, D 14059 Berlin

Holzlöhner, Ulrich, Dr.-Ing., Gartenstraße 5, D 14169 Berlin

Iosifidou, Constantina, Dipl.-Ing., Democritus University of Thrace, Department of Civil Engineering, 12 Vas. Sofias Str, GR 67100 Xanthi

Ittershagen, Marc, Dipl.-Ing., Technische Universität Darmstadt, Institut und Versuchsanstalt für Geotechnik, Petersenstraße 13, D 64287 Darmstadt

Karstedt, Jens, Dr.-Ing., Berlin-Brandendurger Institut für Geotechnik GmbH, Dr.-Ing. Karstedt und Partner, Limastraße 25a, D 14163 Berlin

Katzenbach, Rolf, Univ.-Prof. Dr.-Ing., Technische Universität Darmstadt, Institut und Versuchsanstalt für Geotechnik, Petersenstraße 13, D 64287 Darmstadt

Kausel, Eduardo, Prof. Dr., Massachusetts Institute of Technology, Department of Civil and Environmental Engineering, 77 Massachusetts Avenue, USA 02139 Cambridge, MA 
Klapperich, Herbert, Univ.-Prof. Dr.-Ing., Technische Universität Bergakademie Freiberg, Institut für Geotechnik, Lehrstuhl für Bodenmechanik, bergbauliche Geotechnik und Grundbau, Gustav-Zeuner-Straße 1, D 09596 Freiberg

Kolymbas, Dimitrios, o. Univ.-Prof. Dr. techn., Universität Innsbruck, Institut für Geotechnik und Tunnelbau, Technikerstraße 13, A 6020 Innsbruck

Kramer, Helmut, Prof. Dr.-Ing., Ingenieurbüro Prof. Dr.-Ing. Kramer \& Dipl.-Ing. Albrecht, Glockengießerwall 1, D 20095 Hamburg

Lamers, Jörg, Dipl.-Ing., Ingenieurbüro Prof. Dr.-Ing. Kramer \& Dipl.-Ing. Albrecht, Glockengießerwall 1, D 20095 Hamburg

Li, Xiang-Song, Assoc. Prof. Dr., The Hong Kong University of Science and Technology, Department of Civil Engineering, Clearwater Bay, Kowloon, HK Hong Kong

Linder, Wolf-Rüdiger, Dr.-Ing., Brückner Grundbau GmbH, Am Lichtbogen 8, D 45141 Essen

Liolios, Asterios, Prof. Dr., Democritus University of Thrace, Department of Civil Engineering, 12 Vas. Sofias Str, GR 67100 Xanthi

Mallwitz, Karl, Prof. Dr.-Ing., Fachhochschule Neubrandenburg, Fachgebiet Verkehrsbau, Bodenmechanik, Grundbau, Brodaer Straße 2, D 17033 Neubrandenburg

Mittag, Jens, Dr.-Ing., GuD Geotechnik und Dynamik Consult GmbH, Dudenstraße 78, D 10965 Berlin

Mussehl, Dirk, Dipl.-Ing., Berlin-Brandendurger Institut für Geotechnik GmbH, Dr.-Ing. Karstedt und Partner, Limastraße 25a, D 14163 Berlin

Mylonakis, George, Prof. Dr., University of Patras, Department of Civil Engineering, GR 26500 Patras

Niemunis, Andrzej, Dr.-Ing., Ruhr-Universität Bochum, Lehrstuhl für Grundbau und Bodenmechanik, Gebäude IA-4/126, Universitätsstraße 150, D 44801 Bochum

Pekoll, Oskar-H., Dipl.-Ing., GuD Geotechnik und Dynamik Consult GmbH, Dudenstraße 78, D 10965 Berlin

Pitilakis, Kyriazis D., Prof. Dr., Aristotle University of Thessaloniki, Department of Civil Engineering, P.O.Box 450, GR 54006 Thessaloniki

Rackwitz, Frank, Dr.-Ing., Technische Universität Berlin, Fachgebiet Grundbau und Bodenmechanik, Gustav-Meyer-Allee 25, D 13355 Berlin

Ramm, Hendrik, Dipl.-Ing., Technische Universität Darmstadt, Institut und Versuchsanstalt für Geotechnik, Petersenstraße 13, D 64287 Darmstadt

Richter, Thomas, Dr.-Ing., GuD Geotechnik und Dynamik Consult GmbH, Dudenstraße 78, D 10965 Berlin

Rohrmann, Rolf G., Dipl.-Ing., Bundesanstalt für Materialforschung und -prüfung, Fachgruppe VII.2 - Ingenieurbau, Unter den Eichen 87, D 12205 Berlin

Rücker, Werner, Prof. Dr.-Ing., Bundesanstalt für Materialforschung und -prüfung, Fachgruppe VII.2 - Ingenieurbau, Unter den Eichen 87, D 12205 Berlin 
Schepers, Winfried, Dipl.-Ing., Technische Universität Berlin, Fachgebiet Grundbau und Bodenmechanik, Gustav-Meyer-Allee 25, D 13355 Berlin

Schick, Peter, Dr.-Ing., Technische Universität Berlin, Fachgebiet Grundbau und Bodenmechanik, Gustav-Meyer-Allee 25, D 13355 Berlin

Schneider, Nikolaus, Dipl.-Ing. Univ., GuD Geotechnik und Dynamik Consult GmbH, Dudenstraße 78, D 10965 Berlin

Syngros, Costis, Dr., University of Patras, Department of Civil Engineering, GR 26500 Patras

Treffer, Andreas, Dipl.-Ing., DB ProjektBau GmbH, Niederlassung Süd, Projektzentrum München, Landsberger Straße 287a, D 80687 München

Triantafyllidis, Theodor, Univ.-Prof. Dr.-Ing., Ruhr-Universität Bochum, Lehrstuhl für Grundbau und Bodenmechanik, Gebäude IA-4/126, Universitätsstraße 150, D 44801 Bochum

Trobisch, Steffen, Dipl.-Ing., Berlin-Brandendurger Institut für Geotechnik GmbH, Dr.-Ing. Karstedt und Partner, Limastraße 25a, D 14163 Berlin

Tsotsos, Stefanos, Prof. Dr., Aristotle University of Thessaloniki, Department of Civil Engineering, GR 54006 Thessaloniki

Vrettos, Christos, Univ.-Prof. Dr.-Ing., Technische Universität Kaiserslautern, Fachgebiet Bodenmechanik und Grundbau, Postfach 3049, D 67653 Kaiserslautern

Walz, Bernhard, Univ.-Prof. em. Dr.-Ing., Bergische Universität Wuppertal, Fachbereich D - Abt. Bauingenieurwesen, Lehr- und Forschungsgebiet Unterirdisches Bauen, Grundbau und Bodenmechanik, Pauluskirchstraße 7, D 42285 Wuppertal

Werner, Anke, Dipl.-Ing., Technische Universität Darmstadt, Institut und Versuchsanstalt für Geotechnik , Petersenstraße 13, D 64287 Darmstadt

Wichtmann, Torsten, Dipl.-Ing., Ruhr-Universität Bochum, Lehrstuhl für Grundbau und Bodenmechanik, Gebäude IA-4/126, Universitätsstraße 150, D 44801 Bochum

Yang, Jun, Assist. Prof. Dr., The University of Hong Kong, Department of Civil Engineering, Pokfulham, Hong Kong

Yeroyianni, Maria, Dipl.-Ing., Commission of the European Communities, Rue de la Loi 200, B 1049 Brussels 


\section{Inhalt}

\section{Theoretische und experimentelle Bodenmechanik}

Vibro-Hypoplastizität

G. Gudehus

Fundamentsetzungen unter Gebrauchslast

U. Holzlöhner

Einfluss der Endflächenreibung bei triaxialen Kompressionsversuchen

mit Berliner Sand

F. Rackwitz

Durchführung und Auswertung von Spaltzugversuchen mit kohäsiven Böden

P. Schick

Möglichkeiten und Grenzen bodenmechanischer 1g-Modellversuche

B. Walz

\section{Bodendynamik und dynamische Boden-Bauwerk Interaktion}

Moment mal, wie kann das denn sein, Mr. Lamb?

C. Bode und R. Hirschauer

The Collapse of the Hanshin Expressway (Fukae) Bridge, Kobe 1995:

Soil-Foundation-Structure Interaction, Reconstruction, Seismic Isolation

G. Gazetas, I. Anastasopoulos, N. Gerolymos, G. Mylonakis und C. Syngros

Baudynamische Berechnung eines Produktionsgebäudes.

H. Kramer und J. Lamers

Analysis of Seismic Response of Granular Soil Deposits.

X. S. $\mathrm{Li}$

A Numerical Approach to the Unilateral Contact Dynamic

Problem of Soil-Pile Interaction.

A. Liolios, C. Iosifidou, S. Tsotsos, K. Pitilakis und M. Yeroyianni 
A Method to Increase the Accuracy of the Green's Functions

for Layered Media Obtained by Means of the Thin Layer Method

W. Schepers und E. Kausel

Kumulatives und dynamisches Verhalten von Böden

T. Triantafyllidis, T. Wichtmann und A. Niemunis

Influence of Water Saturation on Seismic Site Amplification

J. Yang

\section{Geotechnik und Spezialtiefbau}

Geotechnische Besonderheiten an ausgewählten Bauvorhaben im

Hamburger Hafen

J. Franke und C. Eickenbrock

Rechnerische und tatsächliche Verformungen tiefer Baugruben am Lehrter Bahnhof in Berlin.

J. Karstedt, S. Trobisch und D. Mussehl

Die Entwicklung neuer Gründungstechnologien am Beispiel

der Forschungs-Kooperation der TU Berlin mit der TU Darmstadt

R. Katzenbach, M. Ittershagen, H. Ramm und A. Werner

Bodenbewehrung - offene Fragen

D. Kolymbas

Probebelastungen zur Ermittlung des Tragverhaltens von Bohrpfählen in Tonstein

W.-R. Linder

Bemessung der Kombinierten Pfahl-Platten-Gründungen (KPP)

nach neuer DIN 1054:2004-08 bzw. DIN 1045-1:2001-07 gezeigt am Beispiel

Hochhauskomplex ,Föderation“" in Moskau-City

O.-H. Pekoll und T. Richter

Ein Näherungsverfahren zur Berechnung von Pfahl-Plattengründungen mit unregelmäßiger Geometrie

C. Vrettos 


\section{Verkehrswegebau und Umweltgeotechnik}

Meßtechnische Ermittlung der Beanspruchungen einer

Festen Fahrbahn unter Zugüberfahrt

S. Bergmann

Verbreiterung eines Bahndammes auf bindigen und organischen Böden

K.-M. Bochert, J. Mittag und A. Treffer

Die Entwicklung der Scherfestigkeit von Dichtwandmassen

als erweitertes Prüfkriterium der Qualitätssicherung.

H.-L. Hebener und N. Schneider

Interdisziplinäres Flächenrecycling - Brachennutzung

contra Grünland-Verbrauch

H. Klapperich

Historische Verkehrsflächenbefestigungen

K. Mallwitz

Experimentelle Untersuchungen zu Schotterfahrwegen auf Brücken

W. Rücker, M. Baeßler und R.G. Rohrmann

Univ.-Prof. Dr.-Ing. habil. Stavros A. Savidis

Lebens- und Berufsweg.

Publikationsverzeichnis

Fest-Kolloquium am 19. November 2004 
1

Theoretische und experimentelle Bodenmechanik 